\title{
Systemic and Myocardial Effects of ATP and Adenosine during Hypoxic Pulmonary Hypertension in Lambs
}

\author{
G. GANESH KONDURI \\ Departments of Pediatrics, Children s Hospital of Michigan, Hutzel Hospital, and Wayne State \\ University School of Medicine, Detroit, Michigan 48201
}

ABSTRACT

The systemic and myocardial effects of adenosine and ATP were investigated in 12 newborn lambs, instrumented at 5-7 d of age with catheters in the aorta, pulmonary artery, coronary sinus, and right and left atria and flow transducers around the main pulmonary artery and left circumflex coronary artery. Studies were done 3-7 d after recovery from surgery. Pulmonary hypertension was induced by exposure to alveolar hypoxia $\left(10 \% \mathrm{O}_{2}, 5 \% \mathrm{CO}_{2}\right.$, and $85 \% \mathrm{~N}_{2}$ ), which was maintained throughout the experiment. Adenosine, ATP, or an equal volume of saline (control) was infused into the right atrial line in doses of 0.04 to $2.5 \mu \mathrm{mol} / \mathrm{kg} / \mathrm{min}$ during hypoxia. Alveolar hypoxia caused significant increases in pulmonary artery pressure, pulmonary vascular resistance, left circumflex flow, left ventricular $\mathrm{O}_{2}$ consumption, and systemic and myocardial $\mathrm{O}_{2}$ extraction and a decrease in systemic $\mathrm{O}_{2}$ transport. ATP and adenosine caused selective decreases in pulmonary artery pressure and pulmonary vascular resistance at doses of 0.04 to $0.30 \mu \mathrm{mol} / \mathrm{kg} / \mathrm{min}$ and decreases in both pulmonary and systemic pressures and resistances at 0.60 to 2.5 $\mu \mathrm{mol} / \mathrm{kg} / \mathrm{min}$. ATP and adenosine caused increases in systemic $\mathrm{O}_{2}$ transport, left circumflex flow, left ventricular $\mathrm{O}_{2}$ transport, and left ventricular $\mathrm{O}_{2}$ consumption and decreases in systemic $\mathrm{O}_{2}$ extraction and left ventricular $\mathrm{O}_{2}$ extraction at 0.3 to $2.5 \mu \mathrm{mol} / \mathrm{kg} / \mathrm{min}$. Systemic $\mathrm{O}_{2}$ con-

Persistent pulmonary hypertension of the newborn is a condition associated with high morbidity and mortality. The affected infants have an increased PVR and hypoxemia secondary to right-to-left shunting of blood across fetal channels (1). Vasodilator drugs that are used in the management of this condition, such as tolazoline, seem to dilate both pulmonary and systemic vessels and cause

Received August 12, 1993; accepted February 1, 1994.

Correspondence and reprint requests: G. Ganesh Konduri, M.D., Department of Pediatrics, Hutzel Hospital, 4707 St. Antoine Blvd., Detroit, M1 48201.

Supported by grants from the American Heart Association of Michigan and the Children's Hospital of Michigan Research Endowment Fund.

'Presented in part at the Society for Pediatric Research meeting, New Orleans, LA, May 1991. sumption did not change during the study. Arterial and coronary sinus blood lactate levels increased during hypoxia and decreased from hypoxia at $2.5-\mu \mathrm{mol} / \mathrm{kg} / \mathrm{min}$ infusion rates of adenosine and ATP. Adenosine and ATP cause selective pulmonary vasodilation in lambs with pulmonary hypertension at doses of 0.04 to $0.30 \mu \mathrm{mol} / \mathrm{kg} / \mathrm{min}$ and improvement in systemic and myocardial $\mathrm{O}_{2}$ delivery at larger doses. Adenosine and ATP may have a beneficial role in the management of pulmonary hypertension in children. (Pediatr Res 36: 41-48, 1994)

\author{
Abbreviations \\ PVR, pulmonary vascular resistance \\ SVR, systemic vascular resistance \\ PAP, pulmonary artery pressure \\ SOT, systemic $\mathrm{O}_{2}$ transport \\ $\mathrm{VO}_{2}$, systemic $\mathrm{O}_{2}$ consumption \\ $\mathrm{V}_{\mathbf{E}}$, systemic $\mathrm{O}_{2}$ extraction \\ MOT, left ventricular $\mathrm{O}_{2}$ transport \\ $\mathrm{MVO}_{2}$, left ventricular $\mathrm{O}_{2}$ consumption \\ $\mathbf{M V}_{\mathbf{E}}$, left ventricular $\mathrm{O}_{2}$ extraction \\ $\mathrm{PaO}_{2}$, arterial $\mathrm{PO}_{2}$ \\ $\mathrm{PaCO}_{2}$, arterial $\mathrm{PCO}_{2}$ \\ $\mathrm{FIO}_{2}$, fraction of inspired oxygen
}

hypotension and tachycardia as side effects (2). The increase in heart rate and systemic vasodilation results in increases in $\mathrm{VO}_{2}$ and $\mathrm{MVO}_{2}$ during hypoxia (3). Newborn infants have a high resting cardiac output and $\mathrm{O}_{2}$ consumption (4) and are limited in their ability to augment SOT during hypoxia (5). Therefore, an increase in $\mathrm{O}_{2}$ consumption during vasodilator therapy may compromise tissue oxygenation in hypoxic infants.

Adenosine and ATP are purine nucleotides that dilate systemic and pulmonary vessels by stimulation of $P_{1}$ and $\mathrm{P}_{2}$ purinergic receptors (6). They are important mediators of $\mathrm{O}_{2}$-induced pulmonary vasodilation in fetal lambs (7). The nucleotides have an extremely short half-life and are cleared during a single pass through the lung (8) when 
they are infused in low doses into the right atrium. ATP infusion causes selective pulmonary vasodilation in doses $\leq 0.15 \mu \mathrm{mol} / \mathrm{kg} / \mathrm{min}$ in newborn lambs with pulmonary hypertension induced by alveolar hypoxia (9) or infusion of thromboxane mimetic U46619 (10) and in newborn piglets (11) during hypoxia. ATP has been shown to cause selective pulmonary vasodilation at doses $\leq 0.15 \mu \mathrm{mol} / \mathrm{kg} / \mathrm{min}$ in children with congenital heart disease and preoperative and postoperative pulmonary hypertension (12). Adenosine causes selective pulmonary vasodilation in newborn lambs during hypoxia at doses $\leq 0.15 \mu \mathrm{mol} / \mathrm{kg} / \mathrm{min}$ (13). Preliminary data (14) suggest that adenosine infusion $(0.1-0.2 \mu \mathrm{mol} / \mathrm{kg} / \mathrm{min})$ improves oxygenation without causing hypotension and tachycardia in newborn infants with persistent pulmonary hypertension. We proposed the hypothesis that ATP and adenosine do not alter the $\mathrm{VO}_{2}$ and myocardial $\mathrm{O}_{2}$ consumption in doses that cause selective pulmonary vasodilation. The objectives of the present experiments were to determine the effects of adenosine and ATP on 1) SOT and $\left.\mathrm{VO}_{2}, 2\right)$ MOT and $\mathrm{MVO}_{2}$, and 3) arterial and coronary sinus lactate concentrations as an index of systemic and myocardial oxygenation. We chose the newborn lamb as a model because its pulmonary circulation has been studied extensively in the past, and its response to hypoxia has been well documented (15). We used chronically instrumented animals for our studies because this allows us to study pulmonary and systemic circulations without the confounding effects of surgery and anesthesia.

\section{METHODS}

Twelve lambs were studied between the ages of 8 and $12 \mathrm{~d}$. Each lamb was studied three times during hypoxia: once each with adenosine, ATP, and normal saline (control). Each of the three experiments was done on a separate day, and the order of experiments was randomized for each animal. The study was approved by the Animal Investigation Committee of Wayne State University (Detroit, MI).

Lambs were brought into our laboratory at $1 \mathrm{~d}$ of age and housed in separate Plexiglas cages. They were trained to feed ad libitum from an artificial feeding system that contained goat's milk (Irish Hills Farm natural goat's milk, Mason, MI). Lambs adjusted well to the feeding system and gained weight at the rate of $50-75 \mathrm{~g} / \mathrm{kg} / \mathrm{d}$ before surgery.

Surgical procedure. Each lamb underwent one sterile surgical procedure at 5 to $7 \mathrm{~d}$ of age. Lambs were given preanesthetic medication consisting of atropine sulfate $(0.01 \mathrm{mg} / \mathrm{kg} \mathrm{s.c.})$ and ketamine $\mathrm{HCl}(10 \mathrm{mg} / \mathrm{kg}$ intramuscularly). The animal's trachea was intubated with a cuffed endotracheal tube, and anesthesia was maintained by ventilating the lungs with $1-2 \%$ isoflurane and $\mathrm{O}_{2}$. Rectal temperature and ECG were monitored continuously, and arterial blood gases were measured periodically during surgery. Core temperature was maintained at or close to $39^{\circ} \mathrm{C}$ with a heating pad. An incision was made in the left groin, and catheters were inserted into the descending aorta and inferior vena cava via the femoral artery and femoral vein. A left lateral thoracotomy was done, and catheters were inserted by direct puncture into the main pulmonary artery and right and left atria. A catheter was also inserted into the coronary sinus via the hemiazygos vein. The coronary sinus in the lamb receives the venous return from left ventricular myocardium exclusively (16). Ultrasonic flow transducers were implanted around the main pulmonary artery (size $12 \mathrm{~S}$, Transonic Systems Inc., Ithaca, NY) and around the left circumflex coronary artery (size 2 R, Transonic Systems Inc.). The ductus arteriosus was identified and ligated, and the catheters and flow transducer cables were tunneled to the lamb's back. The animal was allowed to recover from surgery for at least $3 \mathrm{~d}$ before experiments were done. Cardiovascular function and $\mathrm{O}_{2}$ consumption were shown to return to normal by $3 \mathrm{~d}$ after thoracotomy in lambs (17). Antibiotics (procaine penicillin, 50000 $\mathrm{U} / \mathrm{kg}$, and gentamicin, $5 \mathrm{mg} / \mathrm{kg}$, intramuscularly) were administered on the day of surgery and on each postoperative day until the studies were completed.

Experimental protocol. Each lamb was secured in a sling during the study. The vascular catheters were connected to strain gauge manometers (P23XL, Spectramed Inc., Critical Care Division, Oxnard, CA), and the flow transducer cables were connected to a dual-channel ultrasonic transit time blood flow meter (Transonic Systems Inc.). The mean aortic, pulmonary arterial, left and right atrial pressures, and mean pulmonary and left circumflex coronary blood flow were recorded on a Grass model 7D polygraph (Grass Instruments, Quincy, MA). From these data, we calculated cardiac index as cardiac output $\div$ body weight, PVR index as mean (PAP - left atrial pressure) $\div$ cardiac index in $\mathrm{L} / \mathrm{kg}$, and SVR index as mean (systemic arterial pressure - right atrial pressure) $\div$ cardiac index in $\mathrm{L} / \mathrm{kg}$. Arterial, mixed venous, and coronary sinus blood samples were obtained to measure blood gas tensions and blood $\mathrm{pH}$ on a blood gas analyzer (ABL 30, Radiometer, Copenhagen, Denmark). Blood $\mathrm{Hb}$ concentration $(\mathrm{g} / \mathrm{L})$ and the percentage of saturation of $\mathrm{Hb}$ with $\mathrm{O}_{2}$ were measured with a hemoximeter (OSM 2 , Radiometer). Blood $\mathrm{O}_{2}$ content $\left(\mathrm{mL} \mathrm{O}_{2} / \mathrm{L}\right)$ was calculated (4) as (1.36 $\times \mathrm{Hb}$ concentration $\times \% \mathrm{Hb}$ saturation) $+\left(0.003 \times \mathrm{PaO}_{2}\right)$. SOT was calculated as arterial $\mathrm{O}_{2}$ content $\times$ cardiac index, $\mathrm{VO}_{2}$ as cardiac index $\times$ (arterial-mixed venous) $\mathrm{O}_{2}$ contents, $\mathrm{V}_{\mathrm{E}}$ as $\left(\mathrm{VO}_{2} \div \mathrm{SOT}\right) \times$ 100 , MOT as left circumflex coronary flow $\times$ arterial $\mathrm{O}_{2}$ content, $\mathrm{MVO}_{2}$ as left circumflex flow $\times$ (arterialcoronary sinus) $\mathrm{O}_{2}$ contents, and $\mathrm{MV}_{\mathrm{E}}$ as $\left(\mathrm{MVO}_{2} \div\right.$ MOT) $\times 100$.

Ambient temperature of the study room was maintained at $26-27^{\circ} \mathrm{C}$ during the experiment. Baseline hemodynamic variables were measured, and blood samples were drawn during normoxia when the animals were awake and resting quietly in the sling. The animals were allowed to rest in the sling for at least $15 \mathrm{~min}$ before 
baseline variables were measured. Alveolar hypoxia was then induced by placing a loosely fitting plastic bag over the animal's head and allowing it to breathe a gas mixture of $10 \% \mathrm{O}_{2}, 5 \% \mathrm{CO}_{2}$, and $85 \% \mathrm{~N}_{2}$ at a flow rate of 5-8 L/min. Lambs frequently hyperventilate during hypoxia and lower their $\mathrm{PaCO}_{2}$, which tends to attenuate the pulmonary vascular response to hypoxia (5). We added $5 \% \mathrm{CO}_{2}$ to the gas mixture to maintain a stable $\mathrm{PaCO}_{2}$ at or close to $5.3 \mathrm{kPa}(40 \mathrm{~mm} \mathrm{Hg})$. The flow rate of the gas mixture was high enough to prevent rebreathing of expired gas. Variables were measured after the animals had stable hypoxemia for $10 \mathrm{~min}$. Adenosine, ATP, or normal saline was infused into the right atrial line starting at $0.04 \mu \mathrm{mol} / \mathrm{kg} / \mathrm{min}$. Variables were measured after $10 \mathrm{~min}$. The infusion rate was then doubled every $10 \mathrm{~min}$ to give the animal $0.08,0.15,0.30,0.60$, 1.20 , and $2.50 \mu \mathrm{mol} / \mathrm{kg} / \mathrm{min}$ of adenosine or ATP. Each infusion rate was maintained for $10 \mathrm{~min}$, and hemodynamic variables were measured at the end of the 10-min period. Alveolar hypoxia was continued during the infusion of adenosine, ATP, or saline until the end of experiment. The animal was returned to room air at the end of each study and returned to its cage. Subsequent experiments were done after a $24-\mathrm{h}$ recovery. Arterial, mixed venous, and coronary sinus blood samples were drawn at baseline, during hypoxia alone, and at each infusion rate of adenosine, ATP, or saline to measure blood gas tensions, $\mathrm{Hb}$ concentration, and $\mathrm{Hb} \mathrm{O}_{2}$ saturation. Arterial and coronary sinus blood samples were also obtained for blood lactate levels at baseline, during hypoxia alone, and at infusion rates of $0.04,0.15,0.60$, and $2.50 \mu \mathrm{mol} / \mathrm{kg} / \mathrm{min}$. The infusion rates of ATP and adenosine were chosen to obtain minimal and maximal changes in PVR and cardiac index in newborn lambs as determined from previous studies $(9,13)$. The half-lives of ATP and adenosine in blood are $<10 \mathrm{~s}(9,13)$, and 10 min is sufficient to achieve steady state levels of nucleotide in plasma. The volume of fluid infused during the experiment ranged from 0.04 to $2.5 \mathrm{~mL} / \mathrm{min}$, and the total volume was $50 \mathrm{~mL}$ or approximately $9 \mathrm{~mL} / \mathrm{kg}$ over $1.5 \mathrm{~h}$. The infusion of saline at this volume in control studies did not cause significant hemodynamic changes. Animals were awake and did not show agitation during the study. Most of the animals had a shivering response during the last $30 \mathrm{~min}$ of hypoxia, even though ambient temperature was kept constant.

Drug preparation. Adenosine and disodium salt of ATP (Sigma Chemical Co., St. Louis, MO) were obtained in powder form, and the solutions for i.v. administration were prepared each day by mixing them with sterile normal saline to give a final concentration of adenosine or ATP of $4 \mu \mathrm{mol} / \mathrm{mL}$.

Assay for lactate. Blood samples were collected into tubes with $0.5 \mathrm{~mL}$ of $12 \%$ trichloroacetic acid kept at $0-4^{\circ} \mathrm{C}$. Samples were immediately centrifuged, and the supernatant was separated and stored at $-20^{\circ} \mathrm{C}$. Samples were assayed for lactate by an enzymatic method based on conversion of lactate to pyruvate by lactate dehydro- genase. The reduction of NAD from this reaction causes an increase in absorbance at $340 \mathrm{~nm}$, which was measured by a spectrophotometer (Beckman Instruments, Inc., Fullerton, CA). Assays for all samples were run in triplicate. The concentration of lactate in each sample was measured using a four-point standard curve and expressed as $\mathrm{mmol} / \mathrm{L}$. The intraassay and interassay coefficients of variation for this method were $3-5 \%$ and $5-7 \%$, respectively.

Statistical analysis. Data are expressed as mean \pm SD. Data obtained during normoxia and during hypoxia alone from different days on each lamb were compared by single-factor analysis of variance for repeated measures (18) to determine whether baseline variables and response to hypoxia were similar on different days. Data obtained during control, ATP, and adenosine infusions were compared by two-way analysis of variance for repeated measures; the two factors affecting the outcome were hypoxia and dose of ATP, adenosine, or saline. When significant differences $(p<0.05)$ were found, a Duncan's multiple range test (18) was done to determine which means were different.

\section{RESULTS}

Baseline variables measured on different days and their response to hypoxia were comparable and showed no significant differences. Alveolar hypoxia resulted in significant increases in heart rate, cardiac index, PAP, PVR, left circumflex flow, and $\mathrm{MVO}_{2}$ and a significant decrease in SOT (Tables 1 and 2). $\mathrm{VO}_{2}$ did not change and $\mathrm{V}_{\mathrm{E}}$ and $M V_{E}$ increased during hypoxia. There were no further changes in heart rate, cardiac output, SOT, PAP, and PVR when the animals received normal saline infusion. MOT and $\mathrm{MVO}_{2}$ decreased to baseline values during infusion of saline with ongoing hypoxia (Table 2). Administration of ATP and adenosine caused selective decreases in PAP and PVR at doses of 0.04 to $0.30 \mu \mathrm{mol} /$ $\mathrm{kg} / \mathrm{min}$ (Table 1). Infusion of ATP and adenosine at doses of 0.60 to $2.5 \mu \mathrm{mol} / \mathrm{kg} / \mathrm{min}$ caused decreases in PAP, PVR, aortic pressure, and SVR and an increase in cardiac index (Table 1). SOT, left circumflex flow, MOT, and $\mathrm{MVO}_{2}$ also increased at infusion rates of adenosine and ATP of 0.3 to $2.5 \mu \mathrm{mol} / \mathrm{kg} / \mathrm{min}$ (Table 2). $\mathrm{VO}_{2}$ did not change and $V_{E}$ and $M V_{E}$ decreased at infusion rates of ATP and adenosine of 0.30 to $2.50 \mu \mathrm{mol} / \mathrm{kg} / \mathrm{min}$ during hypoxia.

There were significant decreases in $\mathrm{pH}$ and $\mathrm{PaO}_{2}$ and an increase in $\mathrm{PaCO}_{2}$ during alveolar hypoxia in control, ATP, and adenosine experiments (Table 3). The increase in $\mathrm{PaCO}_{2}$ is probably caused by addition of $5 \% \mathrm{CO}_{2}$ to the inspired gas mixture. The plastic bag was loosely tied around the neck of the lambs, and the flow rate was kept high enough to prevent rebreathing of expired gas. Blood $\mathrm{pH}$ and $\mathrm{PaCO}_{2}$ did not change further during the experiments with ATP or adenosine compared with control studies. $\mathrm{PaO}_{2}$ increased significantly from hypoxia alone at infusion rates of ATP and adenosine of $2.5 \mu \mathrm{mol} / \mathrm{kg}$ / 
Table 1. Systemic and pulmonary vascular effects of ATP, adenosine (Ade) and saline (Control) during alveolar hypoxia (Hyp)*

\begin{tabular}{|c|c|c|c|c|c|c|c|c|c|}
\hline \multirow[b]{2}{*}{ Variable } & \multirow[b]{2}{*}{ Study } & \multirow[b]{2}{*}{ Baseline } & \multirow[b]{2}{*}{ Hyp } & \multicolumn{6}{|c|}{ Infusion rates $(\mu \mathrm{mol} / \mathrm{kg} / \mathrm{min})$} \\
\hline & & & & 0.04 & 0.08 & 0.15 & 0.30 & 0.60 & 2.50 \\
\hline Heart rate (bpm) & $\begin{array}{l}\text { Control } \\
\text { ATP } \\
\text { Ade }\end{array}$ & $\begin{array}{l}188 \pm 22 \\
188 \pm 25 \\
184 \pm 30\end{array}$ & $\begin{array}{l}251 \pm 38 \dagger \\
229 \pm 42 \dagger \\
232 \pm 32 \dagger\end{array}$ & $\begin{array}{l}242 \pm 34 \dagger \\
227 \pm 42 \dagger \\
247 \pm 39 \dagger\end{array}$ & $\begin{array}{l}252 \pm 23 \dagger \\
225 \pm 45 \dagger \\
246 \pm 43 \dagger\end{array}$ & $\begin{array}{l}248 \pm 43 \dagger \\
240 \pm 46 \dagger \\
252 \pm 36 \dagger\end{array}$ & $\begin{array}{l}252 \pm 41 \dagger \\
245 \pm 42 \dagger \\
247 \pm 30 \dagger\end{array}$ & $\begin{array}{l}255 \pm 35 \dagger \\
252 \pm 39 \dagger \\
249 \pm 37 \dagger\end{array}$ & $\begin{array}{l}258 \pm 24 \dagger \\
233 \pm 44 \dagger \\
228 \pm 42 \dagger\end{array}$ \\
\hline $\begin{array}{l}\text { Cardiac index } \\
(\mathrm{mL} / \mathrm{kg} / \mathrm{min})\end{array}$ & $\begin{array}{l}\text { Control } \\
\text { ATP } \\
\text { Ade }\end{array}$ & $\begin{array}{l}170 \pm 32 \\
160 \pm 19 \\
166 \pm 28\end{array}$ & $\begin{array}{l}200 \pm 26 \dagger \\
190 \pm 21 \dagger \\
194 \pm 21 \dagger\end{array}$ & $\begin{array}{l}209 \pm 36 \dagger \\
190 \pm 22 \dagger \\
210 \pm 41 \dagger\end{array}$ & $\begin{array}{l}198 \pm 32 \dagger \\
190 \pm 29 \dagger \\
206 \pm 36 \dagger\end{array}$ & $\begin{array}{l}195 \pm 41 \dagger \\
200 \pm 25 \dagger \\
208 \pm 31 \dagger\end{array}$ & $\begin{array}{l}201 \pm 31 \dagger \\
210 \pm 31 \dagger \\
218 \pm 38 \dagger\end{array}$ & $\begin{array}{l}200 \pm 48 \dagger \\
235 \pm 32 \dagger \ddagger \\
232 \pm 41 \dagger \ddagger\end{array}$ & $\begin{array}{l}196 \pm 41 \dagger \\
230 \pm 31 \ddagger \ddagger \\
235 \pm 32 \ddagger \ddagger\end{array}$ \\
\hline SAP $(\mathrm{mm} \mathrm{Hg}) \S$ & $\begin{array}{l}\text { Control } \\
\text { ATP } \\
\text { Ade }\end{array}$ & $\begin{array}{l}63 \pm 9 \\
66 \pm 6 \\
60 \pm 5\end{array}$ & $\begin{array}{l}69 \pm 10 \\
65 \pm 10 \\
60 \pm 8\end{array}$ & $\begin{array}{l}65 \pm 11 \\
62 \pm 9 \\
59 \pm 10\end{array}$ & $\begin{array}{l}66 \pm 10 \\
61 \pm 10 \\
61 \pm 9\end{array}$ & $\begin{array}{l}69 \pm 9 \\
59 \pm 10 \\
56 \pm 9\end{array}$ & $\begin{array}{l}67 \pm 9 \\
60 \pm 11 \\
54 \pm 9\end{array}$ & $\begin{array}{l}68 \pm 3 \\
56 \pm 9 \dagger \ddagger \\
50 \pm 9 \dagger \ddagger\end{array}$ & $\begin{array}{l}64 \pm 7 \\
54 \pm 9 \dagger \ddagger \\
41 \pm 8 \dagger \ddagger\end{array}$ \\
\hline PAP $(\mathrm{mm} \mathrm{Hg}) \S$ & $\begin{array}{l}\text { Control } \\
\text { ATP } \\
\text { Ade }\end{array}$ & $\begin{array}{l}11 \pm 4 \\
11 \pm 2 \\
10 \pm 3\end{array}$ & $\begin{array}{l}20 \pm 3 t \\
22 \pm 4 t \\
20 \pm 3 t\end{array}$ & $\begin{array}{l}19 \pm 3 \dagger \\
16 \pm 3 \dagger \ddagger \\
15 \pm 4 \ddagger\end{array}$ & $\begin{array}{l}19 \pm 4 \dagger \\
17 \pm 3+\ddagger \\
16 \pm 2 \dagger \ddagger\end{array}$ & $\begin{array}{l}20 \pm 4 \dagger \\
15 \pm 4 \ddagger \\
16 \pm 3 \dagger \ddagger\end{array}$ & $\begin{array}{l}22 \pm 4 \dagger \\
16 \pm 2 \dagger \ddagger \\
16 \pm 2 \dagger \ddagger\end{array}$ & $\begin{array}{l}21 \pm 5 \dagger \\
16 \pm 3 \dagger \ddagger \\
16 \pm 4 \dagger \ddagger\end{array}$ & $\begin{array}{l}24 \pm 6 \dagger \\
16 \pm 3 \dagger \ddagger \\
15 \pm 3 \dagger \ddagger\end{array}$ \\
\hline LAP $(\mathrm{mm} \mathrm{Hg}) \S$ & $\begin{array}{l}\text { Control } \\
\text { ATP } \\
\text { Ade }\end{array}$ & $\begin{array}{l}3 \pm 2 \\
3 \pm 1 \\
3 \pm 1\end{array}$ & $\begin{array}{l}1 \pm 1 \\
1 \pm 1 \\
2 \pm 1\end{array}$ & $\begin{array}{l}2 \pm 1 \\
2 \pm 1 \\
2 \pm 1\end{array}$ & $\begin{array}{l}2 \pm 1 \\
3 \pm 1 \\
1 \pm 1\end{array}$ & $\begin{array}{l}2 \pm 2 \\
2 \pm 1 \\
2 \pm 1\end{array}$ & $\begin{array}{l}2 \pm 1 \\
2 \pm 1 \\
2 \pm 2\end{array}$ & $\begin{array}{l}2 \pm 1 \\
4 \pm 2 \\
3 \pm 1\end{array}$ & $\begin{array}{l}1 \pm 1 \\
3 \pm 1 \\
3 \pm 1\end{array}$ \\
\hline $\begin{array}{l}\text { SVR index } \\
\quad\left(\mathrm{mm} \mathrm{Hg} \cdot \mathrm{L}^{-1} \cdot \mathrm{kg}^{-1} \cdot \mathrm{min}^{-1}\right)\end{array}$ & $\begin{array}{l}\text { Control } \\
\text { ATP } \\
\text { Ade }\end{array}$ & $\begin{array}{l}480 \pm 78 \\
449 \pm 82 \\
433 \pm 76\end{array}$ & $\begin{array}{l}420 \pm 70 \\
400 \pm 72 \\
380 \pm 81\end{array}$ & $\begin{array}{l}480 \pm 82 \\
403 \pm 76 \\
350 \pm 62\end{array}$ & $\begin{array}{l}500 \pm 76 \\
422 \pm 78 \\
348 \pm 71\end{array}$ & $\begin{array}{l}497 \pm 82 \\
370 \pm 82 \\
321 \pm 80\end{array}$ & $\begin{array}{l}520 \pm 96 \\
352 \pm 72 \\
300 \pm 70\end{array}$ & $\begin{array}{l}440 \pm 72 \\
310 \pm 68 \ddagger \\
260 \pm 62 \ddagger\end{array}$ & $\begin{array}{l}420 \pm 90 \\
301 \pm 70 \ddagger \\
214 \pm 76 \ddagger\end{array}$ \\
\hline $\begin{array}{l}\text { PVR index } \\
\quad\left(\mathrm{mm} \mathrm{Hg} \cdot \mathrm{L}^{-1} \cdot \mathrm{kg}^{-1} \cdot \mathrm{min}^{-1}\right)\end{array}$ & $\begin{array}{l}\text { Control } \\
\text { ATP } \\
\text { Ade }\end{array}$ & $\begin{array}{l}88 \pm 20 \\
78 \pm 23 \\
77 \pm 19\end{array}$ & $\begin{array}{l}126 \pm 19 \dagger \\
135 \pm 21 \dagger \\
122 \pm 20 \dagger\end{array}$ & $\begin{array}{l}136 \pm 26 \dagger \\
102 \pm 18 \ddagger \\
100 \pm 18 \ddagger\end{array}$ & $\begin{array}{l}130 \pm 21 \dagger \\
100 \pm 17 \ddagger \\
102 \pm 19 \ddagger\end{array}$ & $\begin{aligned} 127 & \pm 28 \dagger \\
102 & \pm 19 \ddagger \\
91 & \pm 19 \ddagger\end{aligned}$ & $\begin{aligned} 150 & \pm 32 \dagger \\
91 & \pm 18 \ddagger \\
90 & \pm 18 \ddagger\end{aligned}$ & $\begin{aligned} 139 & \pm 36 \dagger \\
82 & \pm 19 \ddagger \\
82 & \pm 20 \ddagger\end{aligned}$ & $\begin{aligned} 139 & \pm 40 \dagger \\
80 & \pm 16 \ddagger \\
76 & \pm 18 \ddagger\end{aligned}$ \\
\hline
\end{tabular}

* Mean \pm SD for $n=12$. SAP, systemic arterial pressure; LAP, left atrial pressure. Hyp data were obtained at FIO of $_{2} 0.10$ and fraction of inspired $\mathrm{CO}_{2}$ of 0.05 .

$\dagger p<0.05$ from baseline.

$\ddagger p<0.05$ from Hyp alone.

$\S$ Conversion factor for SI units: $1 \mathrm{~mm} \mathrm{Hg}=0.133 \mathrm{kPa}$.

Table 2. Effects of ATP, adenosine (Ade), and saline (Control) on oxygen transport during alveolar hypoxia (Hyp)*

\begin{tabular}{|c|c|c|c|c|c|c|c|c|c|}
\hline \multirow[b]{2}{*}{ Variable } & \multirow[b]{2}{*}{ Study } & \multirow[b]{2}{*}{ Baseline } & \multirow[b]{2}{*}{ Hyp } & \multicolumn{6}{|c|}{ Infusion rates $(\mu \mathrm{mol} / \mathrm{kg} / \mathrm{min})$} \\
\hline & & & & 0.04 & 0.08 & 0.15 & 0.30 & 0.60 & 2.50 \\
\hline $\mathrm{SOT}\left(\mathrm{mL} \mathrm{O}_{2} / \mathrm{kg} / \mathrm{min}\right)$ & $\begin{array}{l}\text { Control } \\
\text { ATP } \\
\text { Ade }\end{array}$ & $\begin{array}{l}18 \pm 2 \\
17 \pm 2 \\
18 \pm 2\end{array}$ & $\begin{array}{l}15 \pm 2 \dagger \\
13 \pm 3 \dagger \\
15 \pm 2 \dagger\end{array}$ & $\begin{array}{l}14 \pm 2 \dagger \\
13 \pm 3 \dagger \\
16 \pm 2\end{array}$ & $\begin{array}{l}13 \pm 3 \dagger \\
13 \pm 3 \dagger \\
14 \pm 3 \dagger\end{array}$ & $\begin{array}{l}13 \pm 3 \dagger \\
14 \pm 4 \dagger \\
14 \pm 2 \dagger\end{array}$ & $\begin{array}{l}13 \pm 2 \dagger \\
16 \pm 3 \ddagger \\
18 \pm 2 \ddagger\end{array}$ & $\begin{array}{l}13 \pm 3 \dagger \\
16 \pm 2 \ddagger \\
18 \pm 3 \ddagger\end{array}$ & $\begin{array}{l}15 \pm 2 † \\
16 \pm 2 \ddagger \\
18 \pm 3 \ddagger\end{array}$ \\
\hline $\mathrm{VO}_{2}\left(\mathrm{~mL} \mathrm{O}_{2} / \mathrm{kg} / \mathrm{min}\right)$ & $\begin{array}{l}\text { Control } \\
\text { ATP } \\
\text { Ade }\end{array}$ & $\begin{array}{l}8 \pm 1 \\
7 \pm 1 \\
7 \pm 1\end{array}$ & $\begin{array}{l}8 \pm 2 \\
7 \pm 1 \\
6 \pm 2\end{array}$ & $\begin{array}{l}6 \pm 2 \\
7 \pm 1 \\
6 \pm 2\end{array}$ & $\begin{array}{l}7 \pm 2 \\
6 \pm 2 \\
6 \pm 1\end{array}$ & $\begin{array}{l}7 \pm 2 \\
6 \pm 2 \\
7 \pm 2\end{array}$ & $\begin{array}{l}6 \pm 2 \\
7 \pm 1 \\
6 \pm 1\end{array}$ & $\begin{array}{l}7 \pm 2 \\
9 \pm 2 \\
6 \pm 2\end{array}$ & $\begin{array}{l}7 \pm 2 \\
9 \pm 1 \\
6 \pm 2\end{array}$ \\
\hline$V_{E}(\%)$ & $\begin{array}{l}\text { Control } \\
\text { ATP } \\
\text { Ade }\end{array}$ & $\begin{array}{l}32 \pm 6 \\
29 \pm 3 \\
32 \pm 8\end{array}$ & $\begin{array}{l}40 \pm 6 \dagger \\
43 \pm 6 \dagger \\
41 \pm 8 \dagger\end{array}$ & $\begin{array}{l}40 \pm 9 \dagger \\
44 \pm 4 \dagger \\
42 \pm 8 \dagger\end{array}$ & $\begin{array}{l}41 \pm 7 \dagger \\
40 \pm 6 \dagger \\
35 \pm 8\end{array}$ & $\begin{array}{l}40 \pm 7 \dagger \\
37 \pm 7 \\
34 \pm 9\end{array}$ & $\begin{array}{l}38 \pm 9 \\
32 \pm 6 \ddagger \\
32 \pm 10 \ddagger\end{array}$ & $\begin{array}{l}41 \pm 11 \dagger \\
32 \pm 7 \ddagger \\
31 \pm 10 \ddagger\end{array}$ & $\begin{array}{l}41 \pm 10 \dagger \\
32 \pm 6 \ddagger \\
26 \pm 10 \ddagger\end{array}$ \\
\hline $\mathrm{LCF}(\mathrm{mL} / \mathrm{min})$ & $\begin{array}{l}\text { Control } \\
\text { ATP } \\
\text { Ade }\end{array}$ & $\begin{array}{l}14 \pm 6 \\
16 \pm 5 \\
17 \pm 8\end{array}$ & $\begin{array}{l}35 \pm 8 \dagger \\
41 \pm 12 \dagger \\
39 \pm 10 \dagger\end{array}$ & $\begin{array}{l}30 \pm 9 \dagger \\
30 \pm 12 \dagger \\
44 \pm 17 \dagger\end{array}$ & $\begin{array}{l}25 \pm 6 \dagger \\
29 \pm 11 \dagger \\
43 \pm 16 \dagger\end{array}$ & $\begin{array}{l}25 \pm 6 \dagger \\
40 \pm 13 \dagger \\
55 \pm 16 \dagger\end{array}$ & $\begin{array}{l}28 \pm 11 \dagger \\
55 \pm 16 \dagger \ddagger \\
58 \pm 15 \dagger \ddagger\end{array}$ & $\begin{array}{l}27 \pm 9 \dagger \\
78 \pm 17 \dagger \neq \S \\
63 \pm 18 \dagger \ddagger\end{array}$ & $\begin{array}{l}33 \pm 10 \dagger \\
79 \pm 18 \dagger \ddagger \S \\
63 \pm 16 \dagger \ddagger\end{array}$ \\
\hline $\operatorname{MOT}\left(\mathrm{mL} \mathrm{O}_{2} / \mathrm{min}\right)$ & $\begin{array}{l}\text { Control } \\
\text { ATP } \\
\text { Ade }\end{array}$ & $\begin{array}{l}1.8 \pm 0.5 \\
2.1 \pm 0.6 \\
2.5 \pm 0.9\end{array}$ & $\begin{array}{l}3.1 \pm 0.6 \dagger \\
2.9 \pm 0.6 \dagger \\
4.1 \pm 1.3 \dagger\end{array}$ & $\begin{array}{l}2.0 \pm 0.2 \\
2.4 \pm 0.7 \\
3.9 \pm 1 \dagger\end{array}$ & $\begin{array}{l}2.1 \pm 0.3 \\
2.4 \pm 0.6 \\
3.5 \pm 1.3 \dagger\end{array}$ & $\begin{array}{l}1.9 \pm 0.2 \\
3.2 \pm 1 \dagger \\
4.9 \pm 1.5 \dagger\end{array}$ & $\begin{array}{l}1.8 \pm 0.4 \\
4.6 \pm 1.5 \dagger \ddagger \\
6.3 \pm 2+\ddagger\end{array}$ & $\begin{array}{l}1.8 \pm 0.5 \\
5.9 \pm 2 \dagger \ddagger \\
7.2 \pm 2 † \ddagger\end{array}$ & $\begin{aligned} 2.2 & \pm 0.6 \\
6.6 & \pm 2 \dagger \ddagger \\
7 & \pm 2.1 \dagger \ddagger\end{aligned}$ \\
\hline $\mathrm{MVO}_{2}\left(\mathrm{~mL} \mathrm{O}_{2} / \mathrm{min}\right)$ & $\begin{array}{l}\text { Control } \\
\text { ATP } \\
\text { Ade }\end{array}$ & $\begin{array}{l}0.7 \pm 0.03 \\
0.8 \pm 0.3 \\
1.2 \pm 0.1\end{array}$ & $\begin{array}{l}1.5 \pm 0.2 \dagger \\
1.6 \pm 0.3 \dagger \\
1.8 \pm 0.15 \dagger\end{array}$ & $\begin{array}{r}1 \pm 0.1 \\
1.4 \pm 0.2 \\
1.3 \pm 0.2\end{array}$ & $\begin{array}{l}1.1 \pm 0.2 \\
1.3 \pm 0.1 \\
1.3 \pm 0.1\end{array}$ & $\begin{array}{r}1 \pm 0.1 \\
1.3 \pm 0.3 \\
1.6 \pm 0.2\end{array}$ & $\begin{array}{c}1 \pm 0.2 \\
1.4 \pm 0.4 \dagger \\
2.5 \pm 0.6 \dagger\end{array}$ & $\begin{aligned} 1 & \pm 0.1 \\
2.1 & \pm 0.2 \dagger \\
2.5 & \pm 0.8 \dagger\end{aligned}$ & $\begin{array}{l}1.2 \pm 0.4 \\
2.8 \pm 0.6 \dagger \ddagger \\
2.3 \pm 0.4 \dagger\end{array}$ \\
\hline $\mathrm{MV}_{\mathrm{E}}(\%)$ & $\begin{array}{l}\text { Control } \\
\text { ATP } \\
\text { Ade }\end{array}$ & $\begin{array}{l}33 \pm 9 \\
40 \pm 6 \\
39 \pm 9\end{array}$ & $\begin{array}{l}52 \pm 10 \dagger \\
54 \pm 9 \dagger \\
52 \pm 9 \dagger\end{array}$ & $\begin{array}{l}46 \pm 11 \dagger \\
42 \pm 8 \\
54 \pm 10 \dagger\end{array}$ & $\begin{array}{l}50 \pm 10 \dagger \\
42 \pm 9 \\
45 \pm 7\end{array}$ & $\begin{array}{l}48 \pm 7 \dagger \\
38 \pm 9 \ddagger \\
43 \pm 9\end{array}$ & $\begin{array}{l}52 \pm 11 \dagger \\
28 \pm 8 \ddagger \\
40 \pm 7 \ddagger\end{array}$ & $\begin{array}{l}45 \pm 10 \\
26 \pm 9 \neq \\
35 \pm 9 \neq\end{array}$ & $\begin{array}{l}55 \pm 12 \dagger \\
32 \pm 8 \ddagger \\
36 \pm 9 \ddagger\end{array}$ \\
\hline
\end{tabular}

* Mean \pm SD for $n=12$. LCF, left circumflex flow. Hyp data were obtained at $\mathrm{FIO}_{2}$ of 0.10 and fraction of inspired $\mathrm{CO}_{2}$ of 0.05 .

$\dagger p<0.05$ from baseline.

$\ddagger p<0.05$ from Hyp alone.

$\S p<0.05$ from $0.30-\mu \mathrm{mol} / \mathrm{kg} / \mathrm{min}$ infusion rate of ATP. 
Table 3. Arterial blood gas values, blood pH, lactates, and temperature during hypoxia (Hyp) and infusion of saline (Control), $A T P$, and adenosine (Ade)*

\begin{tabular}{|c|c|c|c|c|c|c|c|c|c|}
\hline \multirow[b]{2}{*}{ Variable } & \multirow[b]{2}{*}{ Study } & \multirow[b]{2}{*}{ Baseline } & \multirow[b]{2}{*}{ Hyp } & \multicolumn{6}{|c|}{ Infusion rates $(\mu \mathrm{mol} / \mathrm{kg} / \mathrm{min})$} \\
\hline & & & & 0.04 & 0.08 & 0.15 & 0.30 & 0.60 & 2.50 \\
\hline $\mathrm{pHa}$ & $\begin{array}{l}\text { Control } \\
\text { ATP } \\
\text { Ade }\end{array}$ & $\begin{array}{l}7.45 \pm 0.04 \\
7.44 \pm 0.04 \\
7.43 \pm 0.04\end{array}$ & $\begin{array}{l}7.39 \pm 0.04 \dagger \\
7.38 \pm 0.05 \dagger \\
7.39 \pm 0.05 \dagger\end{array}$ & $\begin{array}{l}7.37 \pm 0.04 \dagger \\
7.36 \pm 0.05 \dagger \\
7.36 \pm 0.05 \dagger\end{array}$ & $\begin{array}{l}7.39 \pm 0.04 \dagger \\
7.36 \pm 0.05 \dagger \\
7.36 \pm 0.05 \dagger\end{array}$ & $\begin{array}{l}7.36 \pm 0.04 \dagger \\
7.36 \pm 0.05 \dagger \\
7.35 \pm 0.05 \dagger\end{array}$ & $\begin{array}{l}7.35 \pm 0.03 \dagger \\
7.37 \pm 0.04 \dagger \\
7.36 \pm 0.05 \dagger\end{array}$ & $\begin{array}{l}7.36 \pm 0.05 \dagger \\
7.35 \pm 0.04 \dagger \\
7.36 \pm 0.04 \dagger\end{array}$ & $\begin{array}{l}7.36 \pm 0.05 \dagger \\
7.35 \pm 0.06 \dagger \\
7.36 \pm 0.05 \dagger\end{array}$ \\
\hline $\mathrm{PaCO}_{2}(\mathrm{kPa}) \ddagger$ & $\begin{array}{l}\text { Control } \\
\text { ATP } \\
\text { Ade }\end{array}$ & $\begin{array}{l}4.8 \pm 0.4 \\
4.7 \pm 0.3 \\
4.8 \pm 0.4\end{array}$ & $\begin{array}{l}5.6 \pm 0.5 \dagger \\
5.9 \pm 0.5 \dagger \\
5.6 \pm 0.5 \dagger\end{array}$ & $\begin{array}{l}5.9 \pm 0.7 \dagger \\
5.9 \pm 0.7 \dagger \\
5.6 \pm 0.4 \dagger\end{array}$ & $\begin{array}{l}6.0 \pm 0.8 \dagger \\
5.9 \pm 0.7 \dagger \\
5.6 \pm 0.4 \dagger\end{array}$ & $\begin{array}{l}5.9 \pm 0.4 \dagger \\
5.6 \pm 0.5 \dagger \\
5.6 \pm 0.8 \dagger\end{array}$ & $\begin{array}{l}5.9 \pm 0.4 \dagger \\
5.3 \pm 0.4 \\
5.5 \pm 0.4 \dagger\end{array}$ & $\begin{array}{l}5.6 \pm 0.4 \dagger \\
5.6 \pm 0.7 \dagger \\
5.3 \pm 0.4\end{array}$ & $\begin{array}{l}5.9 \pm 0.7 \dagger \\
5.6 \pm 0.4 \dagger \\
5.6 \pm 0.4 \dagger\end{array}$ \\
\hline $\mathrm{PaO}_{2}(\mathrm{kPa}) \ddagger$ & $\begin{array}{l}\text { Control } \\
\text { ATP } \\
\text { Ade }\end{array}$ & $\begin{array}{l}10.9 \pm 0.5 \\
10.5 \pm 1 \\
10.1 \pm 0.8\end{array}$ & $\begin{array}{l}5.1 \pm 0.8 \dagger \\
4.7 \pm 0.7 \dagger \\
4.5 \pm 0.5 \dagger\end{array}$ & $\begin{array}{l}4.8 \pm 0.8 \dagger \\
5.2 \pm 0.7 \dagger \\
4.8 \pm 0.5 \dagger\end{array}$ & $\begin{array}{l}4.5 \pm 0.5 \dagger \\
4.4 \pm 0.4 \dagger \\
4.5 \pm 0.8 \dagger\end{array}$ & $\begin{array}{l}4.4 \pm 0.8 \dagger \\
4.7 \pm 0.7 \dagger \\
4.8 \pm 0.9 \dagger\end{array}$ & $\begin{array}{l}4.8 \pm 0.8 \dagger \\
4.9 \pm 0.7 \dagger \\
5.3 \pm 0.8 \dagger\end{array}$ & $\begin{array}{l}4.7 \pm 0.9+ \\
5.3 \pm 0.8 \dagger \\
5.3 \pm 0.9 \dagger\end{array}$ & $\begin{array}{l}4.8 \pm 0.8 \dagger \\
5.6 \pm 0.7 \dagger \S \\
5.7 \pm 0.7 \dagger \S\end{array}$ \\
\hline $\begin{array}{l}\text { Arterial } \mathrm{Hb} \\
\text { concentration }(\mathrm{g} / \mathrm{L})\end{array}$ & $\begin{array}{l}\text { Control } \\
\text { ATP } \\
\text { Ade }\end{array}$ & $\begin{array}{l}94 \pm 6 \\
90 \pm 7 \\
98 \pm 6\end{array}$ & $\begin{array}{c}98 \pm 5 \\
98 \pm 6 \dagger \\
105 \pm 5 \dagger\end{array}$ & $\begin{array}{c}98 \pm 6 \\
96 \pm 5 \dagger \\
101 \pm 7\end{array}$ & $\begin{array}{l}97 \pm 10 \\
95 \pm 6 \\
99 \pm 6\end{array}$ & $\begin{array}{l}97 \pm 10 \\
90 \pm 4 \\
98 \pm 7\end{array}$ & $\begin{array}{l}96 \pm 5 \\
91 \pm 7 \\
95 \pm 10\end{array}$ & $\begin{array}{l}98 \pm 10 \\
94 \pm 7 \\
97 \pm 7\end{array}$ & $\begin{array}{l}90 \pm 10 \\
88 \pm 10 \\
88 \pm 8 \S\end{array}$ \\
\hline $\begin{array}{l}\text { Arterial lactate } \\
(\mathrm{mmol} / \mathrm{L})\end{array}$ & $\begin{array}{l}\text { Control } \\
\text { ATP } \\
\text { Ade }\end{array}$ & $\begin{array}{l}0.13 \pm 0.04 \\
0.21 \pm 0.06 \\
0.24 \pm 0.08\end{array}$ & $\begin{array}{l}0.30 \pm 0.06 \dagger \\
0.42 \pm 0.1 \dagger \\
0.50 \pm 0.1 \dagger\end{array}$ & $\begin{array}{l}0.30 \pm 0.1 \dagger \\
0.42 \pm 0.2 \dagger \\
0.56 \pm 0.2 \dagger\end{array}$ & & $\begin{array}{l}0.52 \pm 0.2 \dagger \\
0.71 \pm 0.3 \dagger \\
0.61 \pm 0.2 \dagger\end{array}$ & & $\begin{array}{l}0.47 \pm 0.2 \dagger \\
0.52 \pm 0.3 \dagger \\
0.50 \pm 0.2 \dagger\end{array}$ & $\begin{array}{l}0.65 \pm 0.3 \dagger \\
0.36 \pm 0.2 \| \\
0.30 \pm 0.1 \S \|\end{array}$ \\
\hline $\begin{array}{l}\text { Coronary sinus lactate } \\
(\mathrm{mmol} / \mathrm{L})\end{array}$ & $\begin{array}{l}\text { Control } \\
\text { ATP } \\
\text { Ade }\end{array}$ & $\begin{array}{l}0.04 \pm 0.02 \\
0.06 \pm 0.02 \\
0.08 \pm 0.03\end{array}$ & $\begin{array}{l}0.10 \pm 0.04 \\
0.09 \pm 0.03 \\
0.12 \pm 0.04\end{array}$ & $\begin{array}{l}0.16 \pm 0.05 \dagger \\
0.12 \pm 0.04 \dagger \\
0.16 \pm 0.05 \dagger\end{array}$ & & $\begin{array}{l}0.25 \pm 0.1 \dagger \\
0.21 \pm 0.1 \dagger \\
0.24 \pm 0.15 \dagger\end{array}$ & & $\begin{array}{l}0.30 \pm 0.15 \dagger \\
0.18 \pm 0.06 \dagger \\
0.16 \pm 0.1 \dagger\end{array}$ & $\begin{array}{l}0.32 \pm 0.12 \dagger \\
0.10 \pm 0.05 \| \\
0.12 \pm 0.1 \|\end{array}$ \\
\hline Core temperature $\left({ }^{\circ} \mathrm{C}\right)$ & $\begin{array}{l}\text { Control } \\
\text { ATP } \\
\text { Ade }\end{array}$ & $\begin{array}{l}39.4 \pm 0.2 \\
39.4 \pm 0.3 \\
39.6 \pm 0.2\end{array}$ & $\begin{array}{l}39.2 \pm 0.3 \\
39.2 \pm 0.3 \\
39.3 \pm 0.2\end{array}$ & $\begin{array}{l}39.0 \pm 0.2 \\
39.0 \pm 0.2 \\
39.0 \pm 0.3\end{array}$ & & $\begin{array}{l}39.0 \pm 0.3 \\
38.8 \pm 0.2 \\
38.9 \pm 0.2\end{array}$ & & $\begin{array}{l}38.6 \pm 0.2 \\
38.5 \pm 0.2 \dagger \\
38.6 \pm 0.2\end{array}$ & $\begin{array}{l}38.5 \pm 0.3 \dagger \\
38.4 \pm 0.2 \dagger \\
38.5 \pm 0.3 \dagger\end{array}$ \\
\hline
\end{tabular}

* Data are mean $\pm \mathrm{SD}$ for $n=12$. $\mathrm{pHa}$, arterial $\mathrm{pH}$.

$+p<0.05$ from baseline.

$\ddagger$ Conversion factor: $1 \mathrm{kPa}=7.5 \mathrm{~mm} \mathrm{Hg}$.

$\S p<0.05$ from Hyp.

$\| p<0.05$ from $0.15-\mu \mathrm{mol} / \mathrm{kg} / \mathrm{min}$ infusion rate.

min. The increase in $\mathrm{PaO}_{2}$ was caused by an increase in cardiac output and mixed venous $\mathrm{Po}_{2}$, inasmuch as $\mathrm{FIO}_{2}$ was not changed. The mixed venous $\mathrm{PO}_{2}$ increased from $2.8 \pm 0.5 \mathrm{kPa}(21 \pm 4 \mathrm{~mm} \mathrm{Hg})$ at baseline to $3.6 \pm 0.4 \mathrm{kPa}$ $(27 \pm 3 \mathrm{~mm} \mathrm{Hg}$ ) at an infusion rate of ATP or adenosine of $2.5 \mu \mathrm{mol} / \mathrm{kg} / \mathrm{min}$. Blood $\mathrm{Hb}$ concentration increased during alveolar hypoxia before the infusion of ATP and adenosine in study animals. The increase averaged $7 \mathrm{~g} / \mathrm{L}$ and was similar to the increase in $\mathrm{Hb}$ concentration during hypoxia reported by Moss et al. (5). Arterial blood lactate levels (Table 3) increased during hypoxia and remained elevated during infusion of saline. Arterial lactate levels at $2.5-\mu \mathrm{mol} / \mathrm{kg} / \mathrm{min}$ infusion rates of ATP and adenosine were lower than at $0.15 \mu \mathrm{mol} / \mathrm{kg} / \mathrm{min}$. Lactate levels in coronary sinus were lower than in aorta during both normoxia and hypoxia, indicating a net lactate uptake by the myocardium. Coronary sinus lactate levels increased during hypoxia and remained high during infusion of saline. However, lactate levels in coronary sinus during infusion of adenosine or ATP at $2.50 \mu \mathrm{mol} / \mathrm{kg} / \mathrm{min}$ were lower than at the $0.15-\mu \mathrm{mol} / \mathrm{kg} / \mathrm{min}$ infusion rate, consistent with a decrease in $\mathrm{MV}_{\mathrm{E}}$ at these doses of ATP and adenosine. The core temperature decreased in all animals with prolonged hypoxia (Table 3 ) and occurred at the $2.50-\mu \mathrm{mol} / \mathrm{kg} / \mathrm{min}$ infusion rate of saline, ATP, and adenosine. Animals have shown a shivering response at the time of decrease in core temperature. The increase in
$\mathrm{O}_{2}$ delivery during infusion of ATP and adenosine at 0.3 to $2.5 \mu \mathrm{mol} / \mathrm{kg} / \mathrm{min}$ did not restore core temperature to baseline.

\section{DISCUSSION}

Our study provides the evidence that ATP and adenosine, the purine nucleotides, cause selective pulmonary vasodilation in newborn lambs with hypoxic pulmonary hypertension at doses $\leq 0.30 \mu \mathrm{mol} / \mathrm{kg} / \mathrm{min}$. The results confirm the hypothesis that ATP and adenosine do not alter the $\mathrm{VO}_{2}$ and the myocardial $\mathrm{O}_{2}$ consumption at doses that cause selective pulmonary vasodilation. Adenosine and ATP had a favorable effect on tissue perfusion as evidenced by decreases in $V_{E}$ and $M V_{E}$. These data indicate a potential role for continuous infusions of adenosine and ATP in the management of newborn infants and children with pulmonary hypertension.

The decrease in PVR caused by adenosine and ATP in hypoxic lambs was associated with a significant decrease in PAP and was not accompanied by a change in left atrial pressure or pulmonary blood flow at doses $\leq 0.30 \mu \mathrm{mol} /$ $\mathrm{kg} / \mathrm{min}$. The PAP remained significantly lower at doses $\geq$ $0.60 \mu \mathrm{mol} / \mathrm{kg} / \mathrm{min}$, even though the pulmonary blood flow increased. Therefore, the decrease in PVR caused by ATP and adenosine is probably caused by true pulmonary vasodilation (19) rather than by recruitment of un- 
used vascular channels or dilation of pulmonary veins. These results are in contrast to those of other vasodilators such as hydralazine, tolazoline, and diazoxide that cause a decrease in calculated PVR by increasing the flow (19). Heart rate did not change in our study animals during infusion of adenosine and ATP even at doses that caused an increase in cardiac output and a decrease in SVR. Other vasodilators that decrease SVR, such as tolazoline, cause tachycardia $(3,19)$. ATP and adenosine have been reported to have negative chronotropic effects (20) and are clinically used to decrease heart rate in the presence of supraventricular tachycardia (20). Adenosine is thought to exert its negative chronotropic effects by stimulation of $A_{1}$ receptors in heart (21). We did not observe bradycardia during infusion of ATP or adenosine in any of the animals. The favorable effect of adenosine and ATP at doses of 0.60 to $2.50 \mu \mathrm{mol} / \mathrm{kg} / \mathrm{min}$ on $\mathrm{MV}_{\mathrm{E}}$ may be caused by the increase in coronary flow, without an increase in heart rate above control values.

Adenosine has been implicated as a mediator of coronary vasodilation that occurs during hypoxia and exercise (22). Increased plasma and interstitial fluid levels of adenosine have been observed in association with coronary vasodilation caused by hypoxia (23). We have shown that hypoxia causes a significant increase in coronary flow in newborn lambs and that the increase in flow resulted in a significant increase in $\mathrm{O}_{2}$ delivery to the left ventricular myocardium. However, the increase in flow was not sufficient to compensate for the increase in $\mathrm{O}_{2}$ consumption, because $\mathrm{O}_{2}$ extraction and lactate levels in coronary sinus increased during hypoxia. Infusion of ATP or adenosine during hypoxia causes significant additional vasodilation at doses of 0.3 to $2.50 \mu \mathrm{mol} / \mathrm{kg} / \mathrm{min}$. The increase in coronary flow had a favorable effect on MOT and $\mathrm{MVO}_{2}$. This was indicated by decreases in $\mathrm{O}_{2}$ extraction and coronary sinus lactate levels compared with control studies.

The animals in our study did not have a significant decrease in $\mathrm{VO}_{2}$ during hypoxia. The decrease in SOT was compensated for by an increase in $\mathrm{V}_{\mathrm{E}}$. These results are in contrast to the previous observation of a decrease in $\mathrm{VO}_{2}$ during hypoxia in newborn lambs (5). Moss et al. (5) have reported a decrease in $\mathrm{VO}_{2}$ and an increase in lactate levels above $1 \mathrm{mmol} / \mathrm{L}$ during hypoxia caused by exposure to $\mathrm{FIO}_{2}$ of 0.08 in newborn lambs. $\mathrm{VO}_{2}$ and lactate levels did not change significantly at $\mathrm{FIO}_{2}$ of 0.16 or 0.12. Our studies were done at an $\mathrm{FIO}_{2}$ of 0.10 and resulted in $\mathrm{PaO}_{2}$ of $4.7 \pm 0.5 \mathrm{kPa}(35 \pm 4 \mathrm{~mm} \mathrm{Hg})$, which is comparable to the $\mathrm{PaO}_{2}$ reported by Moss et al. at an $\mathrm{FIO}_{2}$ of 0.12 . Our results are therefore consistent with exposure to moderate hypoxia, as reported by Moss et al. Lactate levels in our study did not increase above 1 $\mathrm{mmol} / \mathrm{L}$ during hypoxia. The increase in lactate levels during hypoxia may be caused by increased work of breathing or a decrease in $\mathrm{O}_{2}$ supply to the tissues. Addition of $5 \% \mathrm{CO}_{2}$ to the gas mixture to prevent a decrease in $\mathrm{PaCO}_{2}$ may have increased the respiratory drive and work of breathing in these animals. However, the $\mathrm{PaCO}_{2}$ values did not differ between control, ATP, and adenosine studies. The lactate levels in control animals increased gradually during the $90 \mathrm{~min}$ of hypoxia. The lactate levels during infusion of adenosine and ATP at 2.5 $\mu \mathrm{mol} / \mathrm{kg} / \mathrm{min}$ were lower than in control studies, coinciding with an increase in SOT. Therefore, the elevation in lactate levels was most likely caused by a decrease in $\mathrm{O}_{2}$ supply to the tissues during alveolar hypoxia. Although arterial lactate levels decreased at an infusion rate of ATP and adenosine of $2.5 \mu \mathrm{mol} / \mathrm{kg} / \mathrm{min}$, a decrease in $V_{E}$ was noted at lower doses of ATP and adenosine. These data indicate a lag between optimization of $\mathrm{O}_{2}$ delivery and clearance of lactate from the blood. The coronary sinus lactate levels were lower than arterial values in newborn lambs, indicating a net lactate uptake by the myocardium. This is consistent with previous studies (24) showing lactate uptake by myocardium in both fetus and newborn. The coronary sinus lactate levels increased with hypoxia and remained elevated in the control experiments. Coronary sinus lactate levels have decreased during infusion of adenosine and ATP at doses of 0.30 to $2.50 \mu \mathrm{mol} / \mathrm{kg} / \mathrm{min}$ compared with control values. The change in lactate levels correlate with an increase in $\mathrm{O}_{2}$ transport and decrease in $\mathrm{O}_{2}$ extraction at these doses.

The increase in $\mathrm{PaO}_{2}$ at $2.5 \mu \mathrm{mol} / \mathrm{kg} / \mathrm{min}$ infusion rates of adenosine and ATP was caused by an increase in mixed venous $\mathrm{Po}_{2}(2.8 \pm 0.5$ to $3.6 \pm 0.4 \mathrm{kPa})$ at this dose. The large increase in cardiac output and decrease in $V_{E}$ at this dose of ATP and adenosine probably caused an increase in mixed venous $\mathrm{PO}_{2}$. Arterial $\mathrm{Hb}$ concentration increased during hypoxia, probably by hemoconcentration. This may reflect an attempt to optimize SOT during a period of decreased $\mathrm{O}_{2}$ availability. A similar increase in $\mathrm{Hb}$ concentration during hypoxia was previously reported in lambs (5). The $\mathrm{Hb}$ concentration gradually returned to baseline as hypoxia was continued. The decrease in $\mathrm{Hb}$ may be caused by a dilutional effect of fluid administered during the study. A greater decrease in $\mathrm{Hb}$ concentration was noted in animals during infusion of ATP and adenosine at $2.5 \mu \mathrm{mol} / \mathrm{kg} / \mathrm{min}$ and may be related to generalized vasodilation observed at this dose.

The core temperature in our animals decreased only after prolonged hypoxia. Each experiment lasted a total of $90 \mathrm{~min}$, and the animals were kept at an $\mathrm{FIO}_{2}$ of 0.10 during this time. There were no significant differences between the three experiments in the decrease in core temperature. The increase in $\mathrm{O}_{2}$ delivery and decrease in $\mathrm{V}_{\mathrm{E}}$ in ATP and adenosine groups were not accompanied by an increase in core temperature. It is possible that temperature may have increased if the infusion of ATP or adenosine was continued for a longer period of time. The core temperature decreased by an average of $1^{\circ} \mathrm{C}$ in our lambs. Sidi et al. (25) have previously reported that the decrease in core temperature during hypoxia depends on ambient temperature. They observed a decrease of $2.1^{\circ} \mathrm{C}$ at an ambient temperature of $17^{\circ} \mathrm{C}$ and a decrease of $0.4^{\circ} \mathrm{C}$ at an ambient temperature of $25^{\circ} \mathrm{C}$ in newborn lambs at an $\mathrm{FIO}_{2}$ of 0.09 for $1 \mathrm{~h}$. Our studies were done 
at an ambient temperature of $26^{\circ} \mathrm{C}$, and the hypoxia $\left(\mathrm{FIO}_{2}\right.$ $=0.10$ ) was maintained for longer than $1 \mathrm{~h}$. Therefore, our results on the decline in core temperature are consistent with the results of Sidi et al. at higher temperatures. The lack of a decrease in $\mathrm{VO}_{2}$ during hypoxia in our animals is also consistent with the observation of Sidi et $a l$. in lambs at a higher $\left(25^{\circ} \mathrm{C}\right)$ ambient temperature. The animals in our study have shown a shivering response during the decrease in core temperature, presumably to increase heat production. Inasmuch as $\mathrm{VO}_{2}$ did not decrease, the decrease in temperature is unlikely to be an attempt to sacrifice thermogenesis during decreased $\mathrm{O}_{2}$ supply. Moss et al. (5) speculated that hypothermia in newborn lambs during hypoxia may represent greater heat loss at this age because of increased body surface area. The increase in work of breathing during hypoxia may also cause greater evaporative heat loss from the respiratory tract.

The purine nucleotides seem to exert their vasodilator effects by stimulation of specific receptors present on endothelial and smooth muscle cells of blood vessels. Burnstock (6) proposed that the effects of purine nucleotides are mediated by two types of purinergic receptors. $P_{1}$ receptors are more sensitive to adenosine, act via the adenylate cyclase system, and are inhibited by methylxanthines. $\mathbf{P}_{2}$ receptors are more sensitive to ATP and are not antagonized by methylxanthines. Based on receptor affinity and ligand binding studies, $P_{1}$ receptors have been further classified into $A_{1}$ and $A_{2}$ subtypes; $A_{1}$ receptors inhibit adenylate cyclase and appear predominantly in the heart, where they mediate the negative chronotropic and inotropic effects of adenosine (21). $A_{2}$ receptors are present on the vascular endothelial cells, stimulate adenylate cyclase, and mediate the vasodilator effects of adenosine (21). $\mathrm{P}_{2}$ receptors are also classified into $P_{2 x}, P_{2 y}, P_{2 z}$, and $P_{2 t}$ receptors (21). The relative predominance of these receptors on the cell varies between different organs, with $\mathrm{P}_{2 y}$ receptors being more predominant on vascular endothelial cells. Stimulation of purine receptors on endothelial cells is accompanied by release of endothelium-derived nitric oxide and prostacyclin (26). Both adenosine and ATP have been shown to cause endothelium-dependent vasodilation in newborn (27) and fetal lambs (28). The mechanism of the selective pulmonary vasodilation seen at lower infusion rates of adenosine and ATP is probably a rapid clearance of the nucleotide by the pulmonary vascular endothelium during the first pass. Fineman et al. (10) have shown that infusions of ATP in identical doses into the right atrium and the left atrium cause either pulmonary or systemic vasodilation. We have previously reported (13) that adenosine infusion in hypoxic lambs at doses of 0.01 to 0.15 $\mu \mathrm{mol} / \mathrm{kg} / \mathrm{min}$ causes no change in left atrial adenosine levels, whereas larger doses cause a dose-dependent increase in adenosine levels in the left atrium.

The selective pulmonary vasodilation caused by adenosine and ATP in lower doses may be useful in the management of infants with pulmonary hypertension.
The wide range of doses that seem to be selective for the pulmonary circulation may facilitate better titration of the dose needed for individual patients. The short half-life and rapid clearance of adenosine and ATP by endothelium may allow us to use these drugs in infants with compromised renal function, a situation in which tolazoline therapy seems to be less desirable (29). However, the presence of right-to-left shunts at the level of the foramen ovale or ductus arteriosus in these infants may allow adenosine and ATP to enter the systemic circulation even in low doses and result in systemic vasodilation. We have ligated the ductus arteriosus in the lambs during surgery. The foramen ovale was closed at autopsy in our animals, and this observation is consistent with closure of the foramen by 1 wk of age reported previously by Dawes et al. (30). In addition, the PAP in our lambs was increased by alveolar hypoxia, whereas infants with persistent pulmonary hypertension usually have near-systemic PAP in spite of receiving high $\mathrm{FIO}_{2}$. The response of pulmonary vessels in infants with pulmonary hypertension may also be different from that of newborn lambs with normal pulmonary circulation because of their structural $(31,32)$ and possibly functional differences. These differences should be considered when extrapolating our results to infants with persistent pulmonary hypertension. Additional studies are required to evaluate fully the role of purinergic receptors in cardiopulmonary changes seen during the perinatal period and the usefulness of adenosine and ATP as therapeutic agents in the management of newborn infants with pulmonary hypertension.

\section{REFERENCES}

1. Fox WW, Gewitz MH, Dinwiddie R, Drummond WH, Peckham GJ 1977 Pulmonary hypertension in the perinatal aspiration syndromes. Pediatrics 59:205-211

2. Drummond WH, Gregory GA, Heymann MA, Phibbs RA 1981 The independent effects of hyperventilation, tolazoline and dopamine on infants with persistent pulmonary hypertension. J Pediatr 98:603-611

3. Konduri GG, Dawe EJ 1989 Tolazoline improves systemic and myocardial oxygen transport and oxygen consumption in hypoxemic newborn lambs. Pediatr Res 25:241A(abstr)

4. Lister G, Walter TK, Versmold HT, Dallman PR, Rudolph AM 1979 Oxygen delivery in lambs: cardiovascular and hematologic development. Am J Physiol 237:H668-H675

5. Moss M, Moreau G, Lister G 1987 Oxygen transport and metabolism in the conscious lamb: the effects of hypoxemia. Pediatr Res $22: 177-183$

6. Burnstock G 1990 Overview: purinergic mechanisms. In: Dubyak GR, Fedan JS (eds) Biological Actions of Extracellular ATP. New York Academy of Sciences, New York, pp 1-18

7. Konduri GG, Gervasio CT, Theodorou AA 1993 Role of adenosine triphosphate and adenosine in oxygen-induced pulmonary vasodilation in fetal lambs. Pediatr Res 33:533-539

8. Konduri GG, Theodorou AA, Mukhopadhyay A, Deshmukh DR 1992 Adenosine triphosphate and adenosine increase the pulmonary blood flow to postnatal levels in fetal lambs. Pediatr Res 31:451-457

9. Konduri GG, Woodard LL 1991 Selective pulmonary vasodilation by low dose infusion of adenosine triphosphate (ATP) in newborn lambs. J Pediatr 119:94-102

10. Fineman JR, Crowley MR, Soifer SJ 1990 Selective pulmonary vasodilation with ATP- $\mathrm{MgCl}_{2}$ during pulmonary hypertension in lambs. J Appl Physiol 69:1836-1842

11. Paidas CN, Dudgeon DL, Haller JA, Clemens MG 1988 Adenosine triphosphate: a potential new therapy for hypoxic pulmonary hypertension. J Pediatr Surg 23:1154-1160

12. Soifer SJ, Fineman JR, Brook M, Heymann MA 1992 ATP-Mgcl ${ }_{2}$ produces selective pulmonary vasodilation in children with postoperative pulmonary hypertension. Pediatr Res 31:36A(abstr)

13. Konduri GG, Woodard LL, Mukhopadhyay A, Deshmukh DR 1992 Adenosine is a pulmonary vasodilator in newborn lambs. Am Rev Respir Dis 146:670-676 
14. Konduri GG, Garcia DC, Kazzi NJ, Shankaran S 1993 Adenosine infusion improves oxygenation in infants with persistent pulmonary hypertension of newborn. Pediatr Res 33:219A(abstr)

15. Custer JR, Hales CA 1985 Influence of alveolar oxygen on pulmonary vasoconstriction in newborn lambs versus sheep. Am Rev Respir Dis 132:326-331

16. Downing SE, Lee JC, Taylor JFN, Halloran K 1973 Influence of norepinephrine and digitalis on myocardial oxygen consumption in the newborn lamb. Circ Res 32:471-479

17. Sidi D, Kuipers JR, Heymann MA, Rudolph AM 1982 Recovery of cardiovascular function in newborn lambs after thoracotomy. Pediatr Res 16:705710

18. Winer BJ 1971 Statistical Principles in Experimental Design, 2nd Ed. McGraw-Hill, New York

19. Rich S, Martinez J, Lam W, Levy PS, Rosen KM 1983 Reassessment of the effects of vasodilator drugs in primary pulmonary hypertension: guidelines for determining a pulmonary vasodilator response. Am Heart J 105:119-127

20. Belhassen B, Pelleg A 1984 Electrophysiologic effects of adenosine triphosphate and adenosine on the mammalian heart: clinical and experimental aspects. J Am Coll Cardiol 4:414-424

21. Paton DM, Taerum $T 1990$ A comparison of $P_{1}$ - and $P_{2}$-purinoceptors. In: Dubyak GR, Fedan JS (eds) Biological Actions of Extracellular ATP. New York Academy of Sciences, New York, pp 165-171

22. Berne RM 1980 The role of adenosine in the regulation of coronary blood flow. Circ Res 47:807-813
23. Berne RM, Knabb RM, Ely SW, Rubio R 1983 Adenosine in the local regulation of blood flow: a brief review. Fed Proc 42:3136-3142

24. Fisher DJ, Heymann MA, Rudolph AM 1980 Myocardial oxygen and carbohydrate consumption in fetal lambs in utero and in adult sheep. Am J Physiol 238:H399-H405

25. Sidi D, Kuipers JRG, Heymann MA, Rudolph AM 1983 Effects of ambient temperature on oxygen consumption and the circulation in newborn lambs at rest and during hypoxemia. Pediatr Res 17:254-258

26. Pearson JD, Slakey LL, Gordon JL 1983 Stimulation of prostaglandin production through purinoceptors on cultured porcine endothelial cells. Biochem J 214:273-276

27. Fineman JR, Heymann MA, Soifer SJ 1991 N-nitro-L-arginine attenuates endothelium-dependent pulmonary vasodilation in lambs. Am J Physiol 260:H1299-H1306

28. Konduri GG, Theodorou AA 1992 Nitro-L-arginine attenuates the pulmonary vasodilation caused by adenosine in fetal lambs. Pediatr Res 31:62A(abstr)

29. Ward RM, Kendig JW, Daniel CH 1982 Potentially lethal accumulation of tolazuline in neonates with oliguria. Pediatr Res 16:313A(abstr)

30. Dawes GS, Mott JC, Widdicombe JG 1955 Closure of the foramen ovale in newborn lambs. J Physiol (Lond) 128:384-395

31. Haworth SG, Reid L 1976 Persistent fetal circulation: newly recognized structural features. J Pediatr 88:614-620

32. Murphy JD, Rabinovitch M, Goldstein JD, Reid L 1981 The structural basis of persistent pulmonary hypertension of the newborn infant. J Pediatr 98:962967 